\title{
Road Information Collector Using Smartphone for Measuring Road Width Based on Object and Lane Detection
}

\author{
https://doi.org/10.3991/ijim.v14i02.11530 \\ Surya Michrandi Nasution ${ }^{(凶)}$, Emir Husni, Kuspriyanto, \\ Rahadian Yusuf, Rahmat Mulyawan \\ Institut Teknologi Bandung, Bandung, Indonesia \\ surya.michrandi@gmail.com
}

\begin{abstract}
Change in traffic condition is unpredictable. This increases the need for alternate routes to avoid congestions and other conditions. The high variance of vehicle types in Indonesia complicates routing, rendering alternative routes sometimes become unavailable for a specific condition of vehicles. Our research is to develop an application for Android smartphone to collect road information and to offer alternative routes for motorcycles; this paper focuses on the first part of the task. The needed information to acquire is road width, so the drivers could use proper alternative routes for their vehicles (e.g. small car or motorcycles). This research uses both object detection and lane detection methods for obtaining road width, and it is quite simple when lane boundaries are detected in road image. When the lane boundaries are not detected, road width is obtained using a vanishing point method. The average error rate for road width measurement using object and lane detection is $19.71 \%$. Meanwhile, the average error rate when there is no lane boundary is in the range of $10-15 \%, 8$ $18 \%$, and $10-19 \%$ for various capturing sides. Reclassification of the road is done when the error rate of road width is set. Accuracy of road category reclassification is in the range of $70-75 \%$ in various sides.
\end{abstract}

Keywords-Road Information, Road Width, Object Detection, Lane Detection, Vanishing Point

\section{$1 \quad$ Introduction}

Traffic demands changes over time, and it is making road condition congested everywhere [1]. Not only that it changes when rush hour is coming, but also when there are events, accidents, or other conditions. Sometimes, to reach a destination, alternate routes are needed to avoid congestion (or other situations). Meanwhile, those routes do not have the same width with common roads. In Indonesia, there are several alternate routes usable only for motorcycle. This condition will become a problem when a car is guided into a road category that does not fit its size.

The variance of vehicle's type is very common in Indonesia. Lubis [2] stated that Indonesia is the third biggest country which has motorcycles after China and India. In 
2016, the Bureau Statistic of Indonesia stated that the amount of motorcycle is dominating other kinds of vehicles [3]. Motorcycle has a small size and could maneuver easily, so it will be the ideal kind of vehicle to drive in a city [4]. This high amount of motorcycle also gives an impact to the traffic condition. That is why Minh et al. [5] stated that motorcycle is one of the reasons traffic jam happened.

This paper will discuss on a system for road information collecting and road width measurement. The system aims to give proper alternate routes based on the vehicle's type. The process uses a crowdsourcing approach by using the application in a driver's smartphone which will capture road situation and traffic condition during the trip. The usage of smartphone is considered because its popularity is growing fast; everyone, even children, in this world has smartphone [6]. Smartphone also easily connects to cloud system, so the information that has been gathered will be stored in a server easily. Soon after, those informations will be extracted for defining a road category.

On the main road, it is common that a boundary line exists for each lane in the road. It will be different in alternate routes, where several roads do not have boundary line. Usually, roads will be divided into several categories such as big, medium, small road and alley. Collected information will be processed by using a machine learning approach.

Machine learning approach will also be used to detect objects and lines that appear in the information. These objects will be limited for a common object such as car and motorcycle with a certain degree of probability. Lane detection approach will be used to detect line marker in the road. For roads that do not have a lane boundary, we will categorize it by using vanishing point concept.

The remainder of this paper is organized as follows. In Section II, the literature review is presented. In Section III, the design and implementation are provided. Section IV discussed the testing of the proposed system. Concluding remarks are given in Section V.

\section{$2 \quad$ Literature Review}

In this research, we implemented road information collector and measured the road width by using object and line detection approach for common roads that had lane boundary. We also tried to measure the width of alternate roads by using vanishing point approach. It is used for measure road which did not have lane boundary.

\subsection{Object detection}

Object detection approach using deep learning method usually use the two-stages detector, such as R-CNN and Fast R-CNN, where it can give high accuracy [7]. The two-stage detector had weakness in significant computational resource and time.

Hoang, et al. [7] stated that the steps that occur in a two-stage detector start with defining region of interest (ROI) and creating a proposed network, continue with classifying in candidate regions. Nowadays, the state-of-the-art method is a one-stage 
detector that could detect an object faster by running a classification step only, but it gives worse accuracy than two-stage detector.

Common methods that used in the one-stage detector are Single Shot Detector (SSD), You Only Look Once (YOLO), and RetinaNet. RetinaNet built based on several one-stage characteristics, such anchors concept in RPN, and feature of pyramid concept in SSD and FPN. Commonly, every detection method is tested using Common Object in Context (COCO) dataset due to achieve its accuracy. Dectection accuracy using RetinaNet could be compared with two-stage detector method [8].

The usage of object detection is to know road situation, such as number of cars, motorcyles, and other type of vehicles in the road. Nasution et al. stated that there is common width of vehicle, such as car and motorcycle which has 1.66 and $0.64 \mathrm{~m}$ [3]. This width is used to compared road width in the road.

\subsection{Lane detection}

Yan and Li [9] stated that there are several characteristics in road image, such as target area that always appears in half-bottom of an image, higher variance of gray than the background, etc. Colour of gray and its variance could have almost the same value, but the edge of the target is the most crucial point in the lane detection process.

Lane detection could be done by implementing canny edge detection [10-12]. In its process, an image will be normalized based on its histogram. Commonly, there will be a color conversion from RGB image into a grayscale image. The grayscale image will be blurred with gaussian blur due to detecting edge easier. Canny edge detection method will search high-value differences between pixels in an image. Finding this value in an RGB image will be more difficult than in a grayscale image.

After new histogram of an image obtained, the process will continue to define ROI. By arranging ROI, the complexity of road lane detection will be minimalized [13]. ROI could be done by removing part of an image except for part of the road. After that edge detection process will find the lane boundary. Normally, there will be two edges (left and right lane boundary). The process will continue with hough transform for drawing the boundary lanes on image. Slope measurement will be done to differentiate the left and right lane [14].

Distance between left and right lane will be measured in order to define the road width in pixel size. This value will be combined with object detection result and it will be converted to real width.

\subsection{Vanishing point}

Vanishing point is a concept used to illustrate in perspective images. It could be built from several lines that gather around one intersection point [15]. From all lines that drawn to the intersection point, several lines illustrate a roadway [16]. The intersection point is not defining the center of the image but illustrates that this point has the farthest depth in perspective. A vanishing point is an infinite point that drawn in one point and commonly appears in the horizon. This method is common used in unstructured road which there are no lane boundaries[17]. 
This approach is used when there are no lane boundaries in the road, especially in alternative road. It will be used to create an illustration of lane boundaries by defining left, center, and right vanishing point in the road.

\section{Design and Implementation}

In this research, we propose a road information collector using Android application which is used to capture the situation of the road. In every informations that collected, road width measurement will be conducted. Lane boundaries in main road are often easy to see, however it is different for alternative road. Due to this, the system will measure the road-width based on object and lane detection (when there are lane boundaries in the road) and vanishing point approach (when there are no lane boundaries in the road). Our proposed system is divided into two parts: first is information gathering using Android application; and information extraction to define road width.

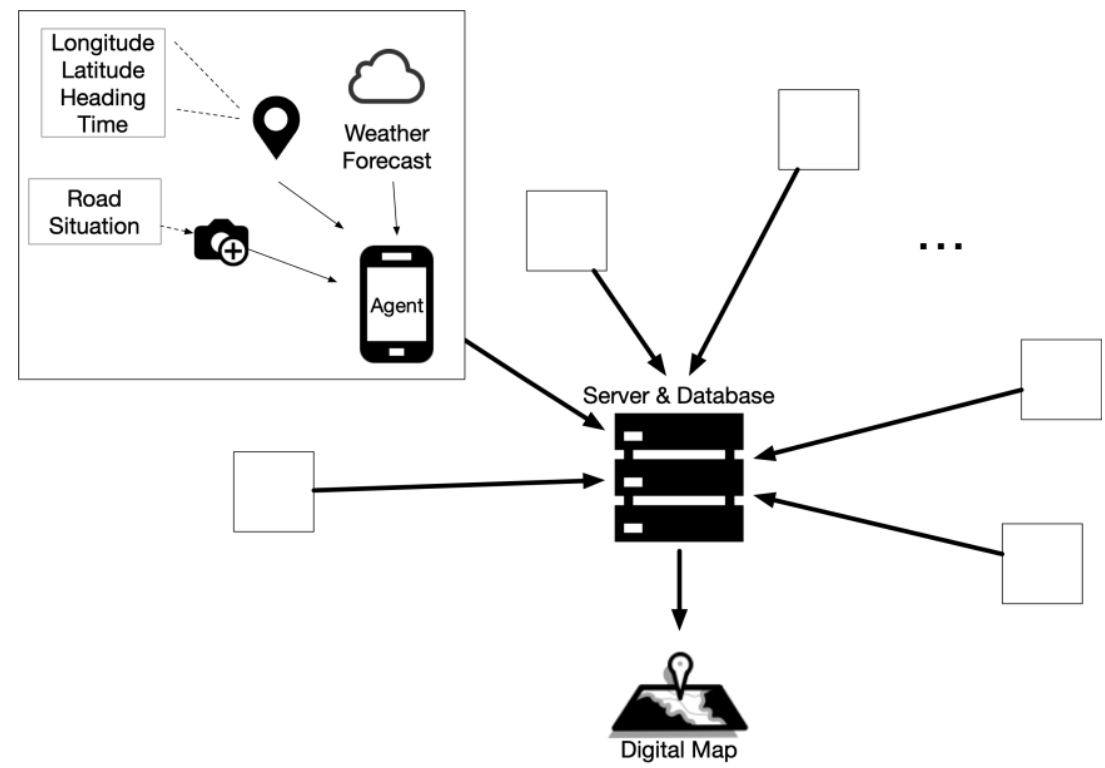

Fig. 1. Design of Proposed System

As mention before, road situation will be massively gathered using smartphone application from several users and store it to a server. Fig. 1 illustrates the design of proposed system. Several road informations collected such as coordinates (longitude and latitude), heading, timestamp, weather, total car and motorcycle, and road image will be collected periodically along the trip. Fig. 2 illustrates the workflow of the system that proposed. 


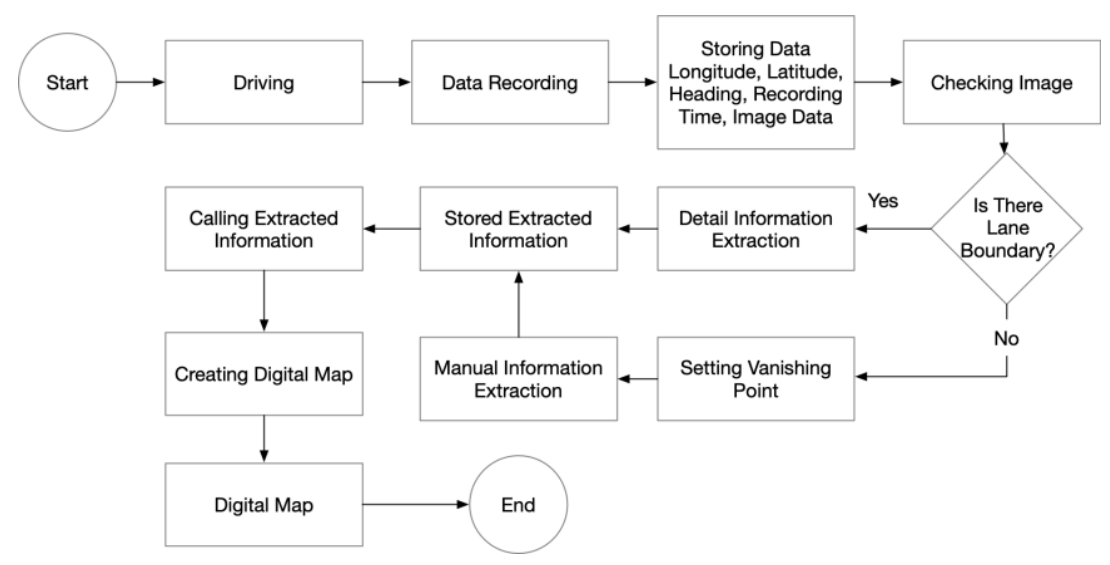

Fig. 2. Workflow of Proposed System

\subsection{Information gathering}

Smartphone will gather information when activated by drivers and work until the driver stop the application. Fig. 3 illustrates the process of collecting information, where smartphone will be attached to a motorcycle.

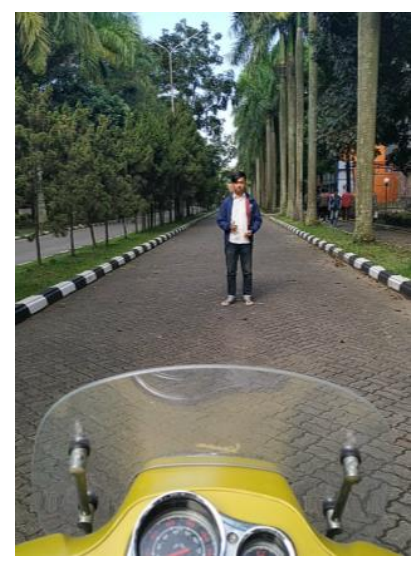

Fig. 3. Process of Collecting Information

Main result acquired from information gathering are coordinates (longitude and latitude), heading, timestamp, and weather condition. These data also collected by using several sensors available in smartphone such as GPS and accelerometer. Weather conditions will be obtained from OpenWeather (open API weather report).

Detailed workflow of the proposed system is designed using Unified Modeling Language (UML) such as sequence, class, and activity diagram. The system works when a driver starts the application, and it will collect information continuously. Driver will execute application, and it will start to request weather report, access GPS 
and camera module. After these primary data collected, it will be stored in a database. This whole process is shown in Fig. 4.

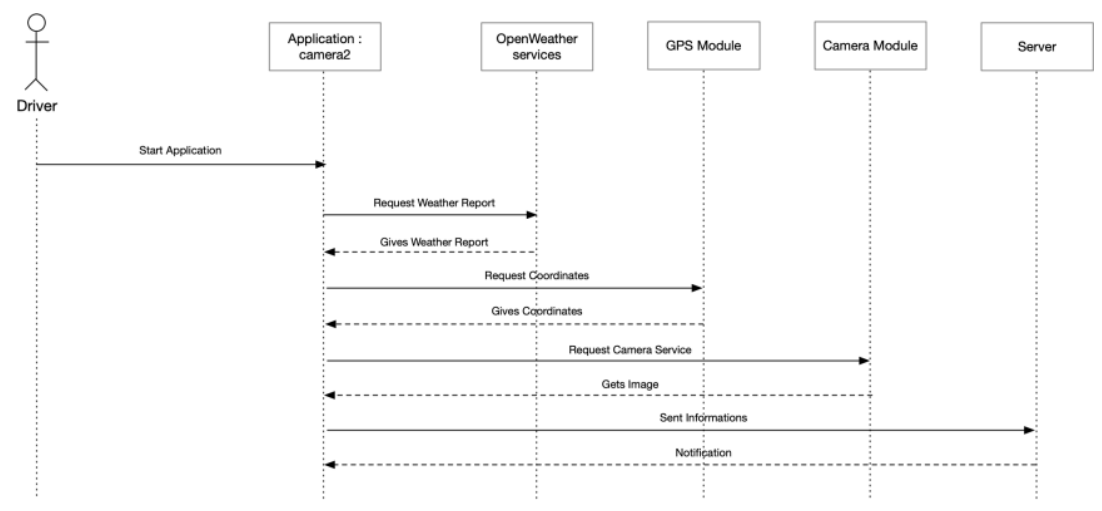

Fig. 4. Sequence Diagram

Class diagram shows relationship between classes used in this application. In this system, we used four main classes (MainActivity, DataCapture, CameraService, and Location). MainActivity is the main class of this application which allows permissions that needed by Android smartphone. Main function of class DataCapture is for collecting coordinates, weather, and heading. Detailed process of resolving coordinates will be conducted in class Location. CameraService is not only used to activate and run camera service, but also building communication with the designated server. Fig. 5 illustrates Class Diagram for the proposed system.

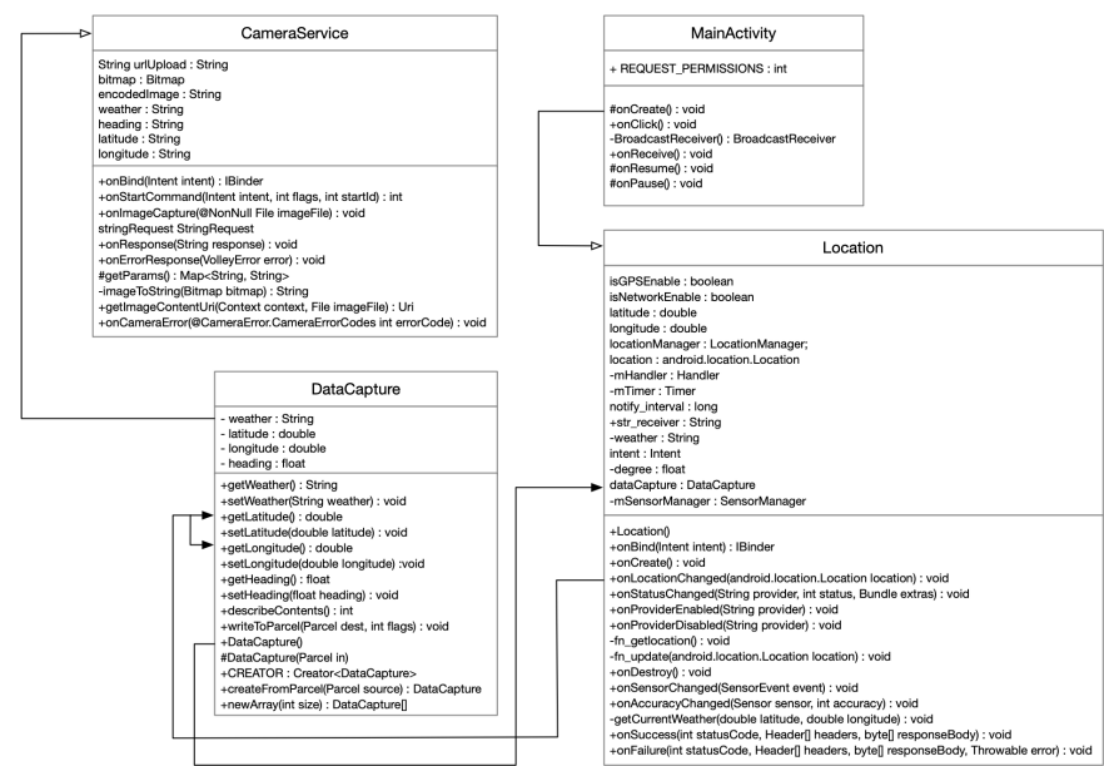

Fig. 5. Class Diagram 
Based on its activity, this proposed system will have four actors (driver, server, database, and user). In the beginning, driver will start their journey and bring a smartphone which will capture road situation periodically. Data will be stored into a database in a server. Those data will be used when there is a request from users. Server uses all data in database to show it in digital map. Fig. 6 shows activity diagram for the proposed system.

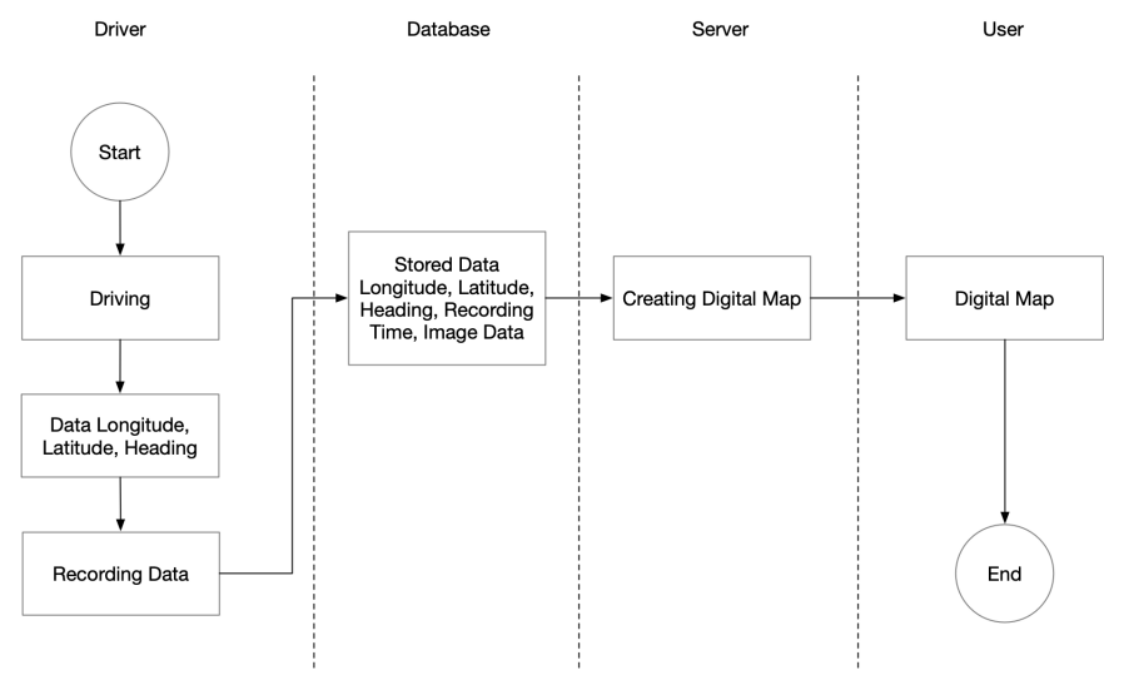

Fig. 6. Activity Diagram

\subsection{Information extraction}

Normal roads: Detection system is needed to extract information to recognize road situation. It can detect common objects on the road, such as car and motorcycle. Detection method in this proposed system uses ImageAI library with RetinaNet for COCO. Fig. 7 shows the detection result in a main road. It detects several common vehicles like car, but it could not detect uncommon vehicles such as truck and pickup.

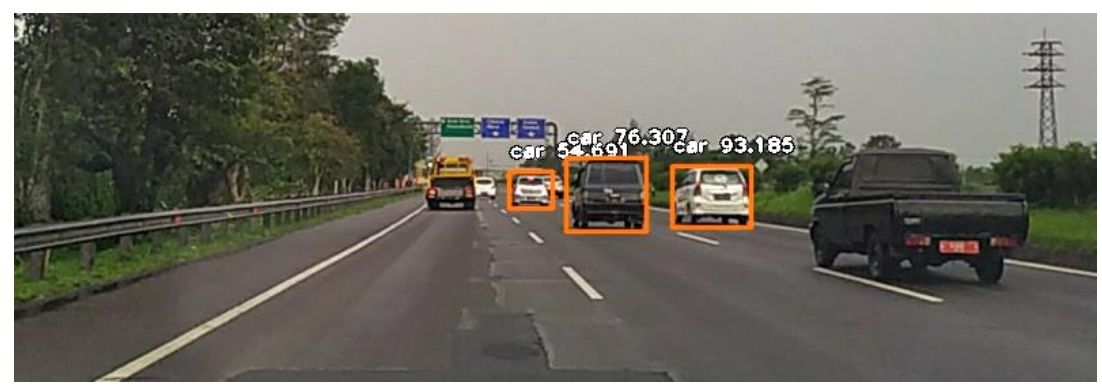

Fig. 7. Vehicle Detection Result 
This proposed system detects not only vehicles but also lane (for normal roads with lane boundaries). The detection is implemented using edge detection. Lane boundaries have high color differentiation with road color so that it will be detected as the edge in road image. Both left and right lane boundaries will be extended until there is an intersection point between those lines. Lane detection result is shown in Fig. 8.

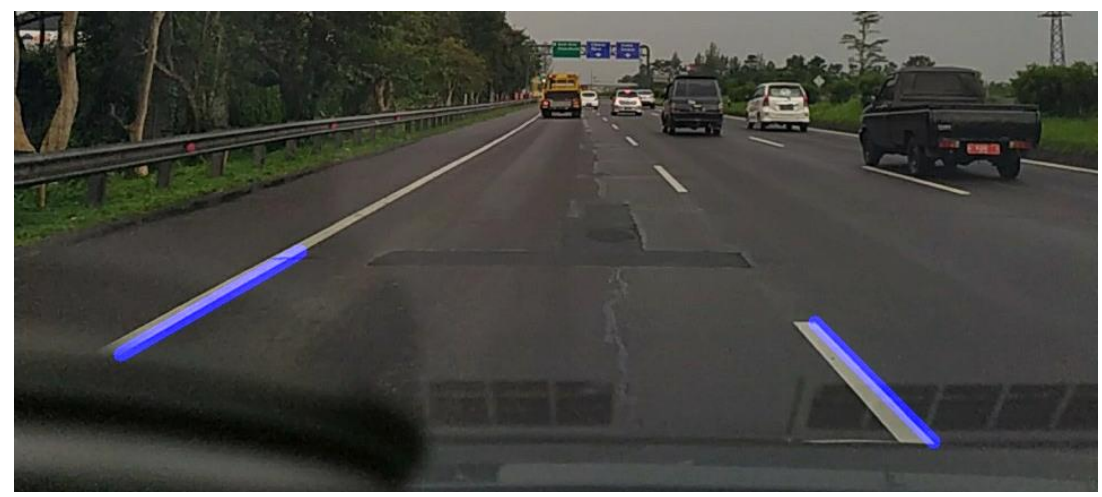

Fig. 8. Lane Detection Result

Both object and lane detection will be combined in order to acquire the average road width. Every detected vehicle will be given a bounding box, and its bottom line is considered as vehicle width. Road width will be obtained from the intersection between bottom line of vehicle's box, left and right lane boundaries. Road width is the distance between intersections of lane boundaries. Fig. 9 shows intersection points between left and right line.

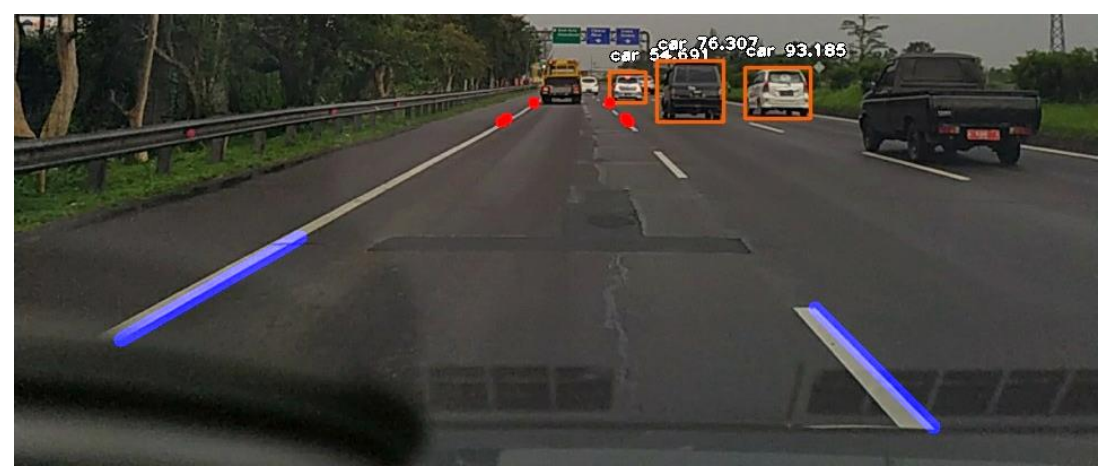

Fig. 9. Combination of Object and Lane Detection

Nasution stated that [2] there is common width for car and motorcycle, $1.66 \mathrm{~m}$ and $0.64 \mathrm{~m}$ respectively. Based on that, road width will be equivalent with common width of vehicles. From all detected vehicle, average road width will be obtained. Distance between left and right intersection points in Fig. 9 are 3.476, 3.179, and 3.005 m. Fig. 10 shows the road width calculation result between intersection points for each de- 
tected vehicle. Average road width ("Lebar Jalan") is $3.22 \mathrm{~m}$ with three detected vehicles.

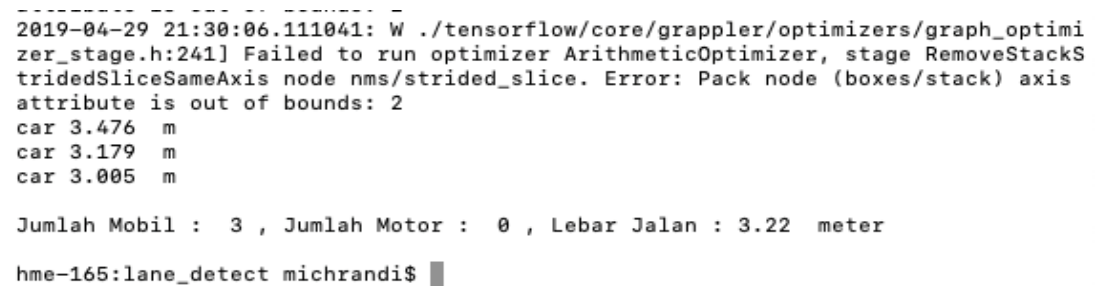

Fig. 10. Average Road Width Calculation

Alternate road: In alternate road, lane boundaries are rarely seen. For dealing with this problem, the calculation will be done manually. It will use three vanishing points seen in the road image : left lane, right lane, and horizon vanishing point. Before we determine road width, we must define the reference of pixel size. This value will be used to measure other roads.

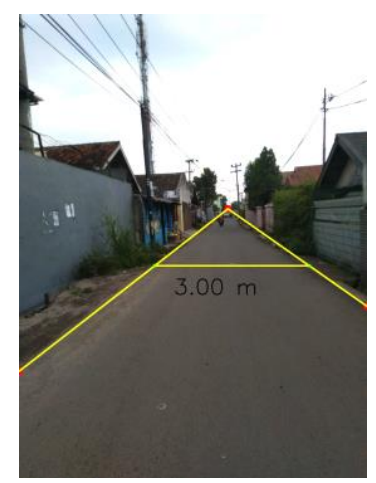

Fig. 11. Road Width without Lane Boundaries

As mentioned previously, position of vanishing points must be determined in road images. Line will be drawn from left or right vanishing point to the horizon. Fig. 11 above shows three vanishing points and two lines that drawn between vanishing points. Distance between two intersections also will be calculated using the same concept. Real width of this road is $3 \mathrm{~m}$ with 426 pixels. Based on this calculation, 1 pixel equals to $0.007 \mathrm{~m}$. This value will be used as a comparative value for other road images.

As seen in Fig. 12, road images (alley and big road) are tested using those value. White line is used for marking the reference lines, and yellow lines is used for road boundaries. Width of the road will be calculated using new intersection points based on new road images. Comparative value is used to recalculate the width of other roads. In our sample, those roads have width $1.28 \mathrm{~m}$ for an alley and $4.14 \mathrm{~m}$ for the big road. 

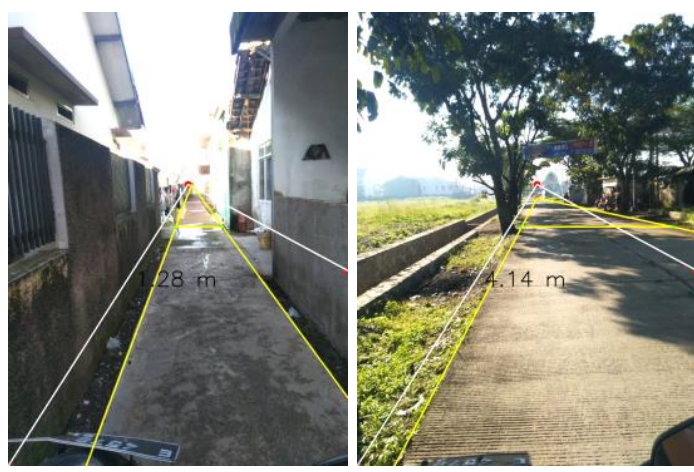

Fig. 12. Measurement of Road Width using Comparative Value

\section{$4 \quad$ Testing}

In this chapter, we test our proposed system in order to know how accurate the result of road measurement by calculating the error rate using Mean Average Percentage Error (MAPE), shown in equation 1. There are several roads that lane boundaries that its width will be calculated using object and lane detection, and there are more than 250 real images without lane boundaries that its width will be calculated using vanishing point. In the end, based on its error rate we tried to reclassify the type of roads.

$$
\operatorname{MAPE}\left(\mathrm{f}, \mathrm{f}^{\prime}\right)=\frac{1}{n} \sum_{i=1}^{n} \frac{\left|f_{i}-f_{i}^{\prime}\right|}{f_{i}}
$$

\subsection{Road width measurement with object and lane detection}

Testing in this method is done by detecting vehicles and comparing between vehicle's width and road width in pixels. Length between two intersection points in the same height with vehicle will be considered as road width. By using equation 2, road width will be obtained by comparing pixel length of road width and vehicle width.

$$
\text { RoadWidth }=\text { VehicleWidth } * \frac{\text { Road Width }(p x)}{\text { Vehicle Size }(p x)}
$$




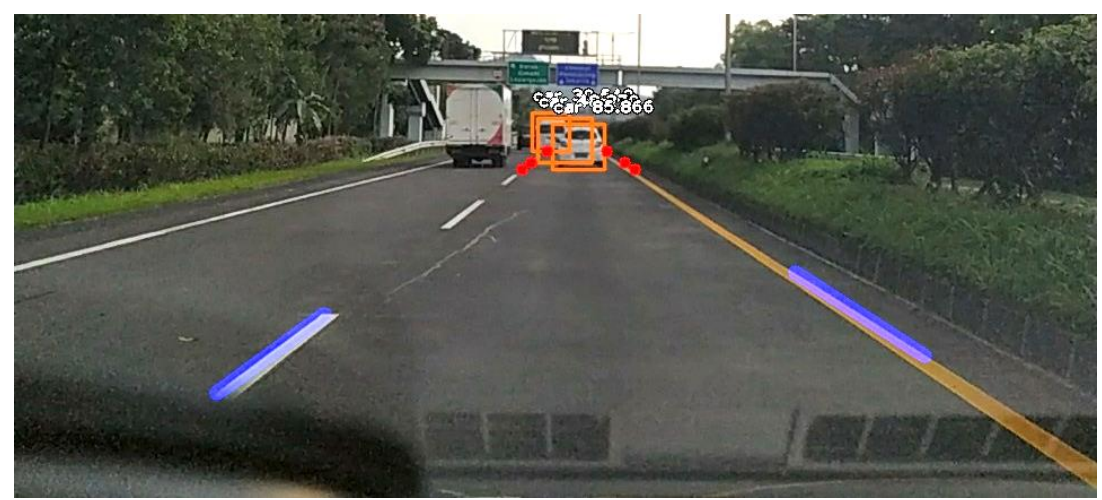

Fig. 13. Testing Result

Fig. 13 shows calculation on a road. Road width result in this testing are $2.66,2.87$ and $3.61 \mathrm{~m}$ from every vehicle that detected and it made the average road width is $3.05 \mathrm{~m}$. Average road width will be used as a comparison to find the road width detection error rate.

In this testing scenario, we test in several roads that have width of $3.5 \mathrm{~m}$. Average error rate acquired is $19.71 \%$ with average width differentiation is $69 \mathrm{~cm}$. This value is acceptable since road width is predicted between 2.81 to $4.19 \mathrm{~m}$ which it still can be used for all type of vehicles. Fig. 14 shows the error rate of road width detection which conducted for 17 roads.

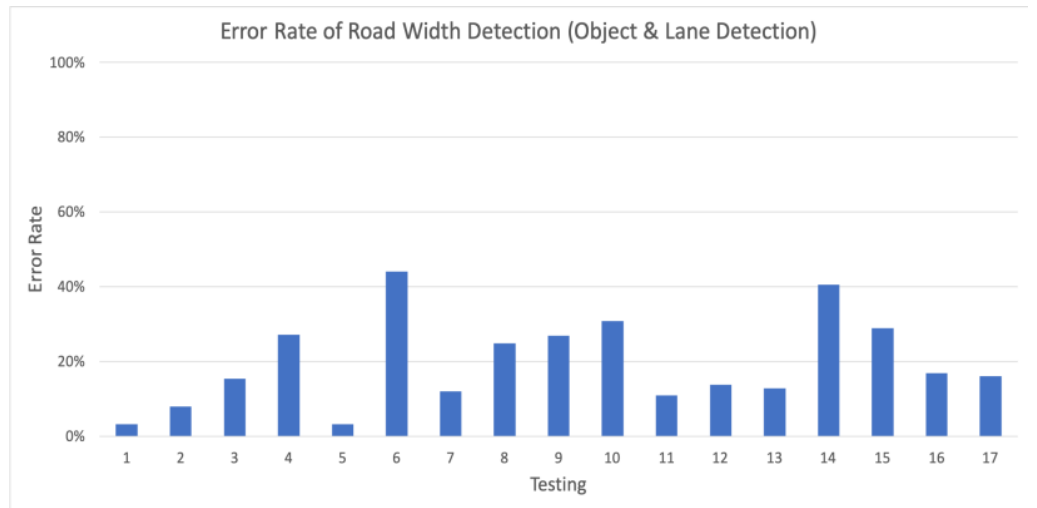

Fig. 14. Error Rate of Road Width Detection

\subsection{Road width measurement with vanishing point}

In the scenario that the lane boundaries are not visible, we classify roads based on its width. Roads will be classified into four types, which are Alley, Small Road, Medium Road, and Big Road. This classification is based on equation 3. 


$$
\text { Roads }=\left\{\begin{array}{c}
\text { Alley, width } \leq 2 m \\
\text { Small Road, } 2 m<\text { width } \leq 3 m \\
\text { Medium Road, } 3 m<\text { width } \leq 4 m \\
\text { Big Road, width }>4 m
\end{array}\right.
$$

Smartphone's camera must be set on stable position perpendicular to the road before determining road width. Real distance is marked in road image. Fig. 15 shows distance between camera with several points in distance from 2 to $5 \mathrm{~m}$, but in this testing scenario we used $3 \mathrm{~m}$ as the distance range. This value is chosen because it gives the best pixel comparison from collected data.

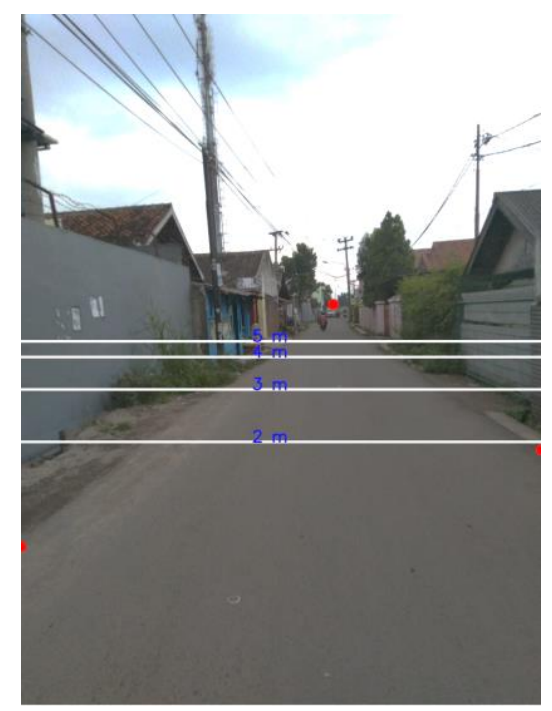

Fig. 15. Range Setting Between Camera and Several Points
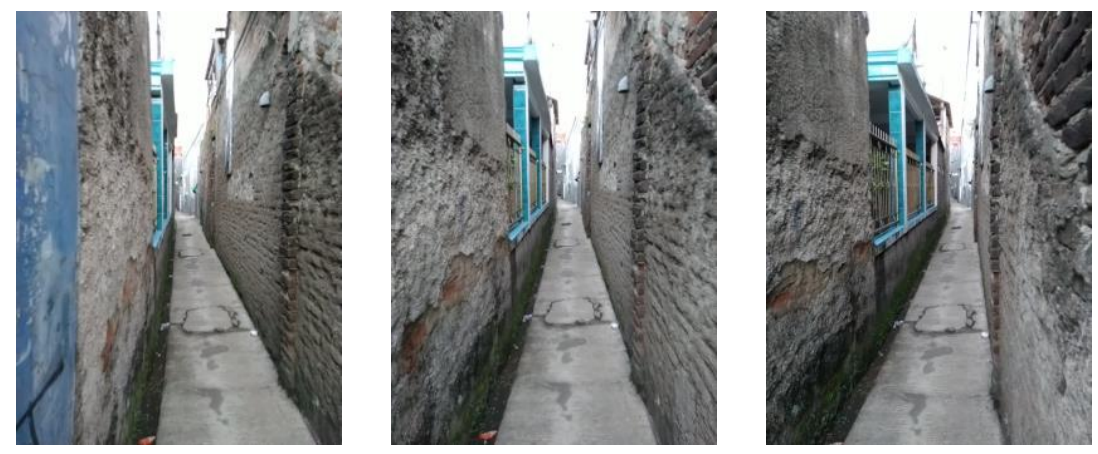

Fig. 16. Various Sides of Road Image: Left, Center, and Right

Roads will be captured from various sides (left, center, and right side), as shown in Fig. 16, in order to get error rate when capturing road image. There is also a probability of pitch changing when capturing road images and it will give a different position 
of vanishing point. To avoid it, normalization of vanishing point must be done by using equation 4. $v p_{\text {diff }}$ is the difference in distance between vanishing points and it also used in equation 5 as references to determine new margin value of lane.

$$
\begin{gathered}
v p_{\text {diff }}=v p_{\text {test }}-v p \\
{\text { laneboundary } y_{\text {new }}}={\text { laneboundary }+v p_{\text {diff }}}^{\text {landata }}
\end{gathered}
$$

The sample result of this method is shown in Fig. 17 where an alley had width 0.93 $\mathrm{m}, 1.1 \mathrm{~m}$, and $0.92 \mathrm{~m}$ for left, center, and right sides respectively. Road width value is obtained using equation 6 , which calculate the distance between 2 points and compare with meter/pixel references.

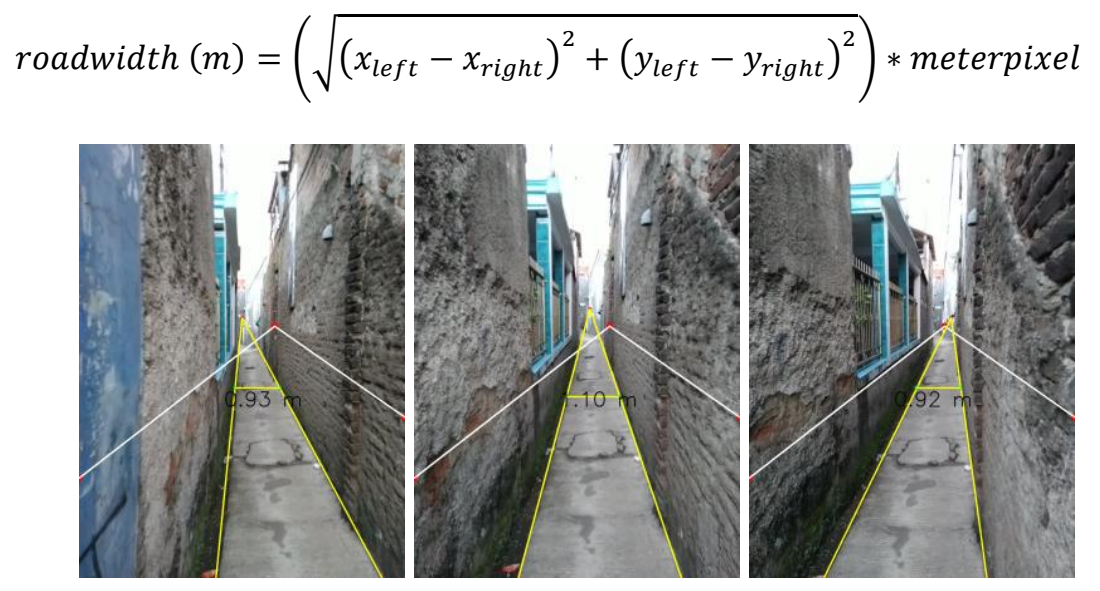

Fig. 17. Road Width for Each Sides

We collected more than 250 road images to test our method. For each road category, there are $24,20,18$, and 24 roads tested for alley, small, medium, and big road respectively. Error rate is obtained from measuring the difference between real width and result of road width calculation. We divide our testing into three parts, error rate measurement for alley and small road, medium and big road, and average error rate for every type of roads and sides.

Error rate for alley and small road: Table 1 shows some sample result of errorrate measurement in alley and small road category. The Left, Center, and Right side of alley road category has the result range of $0.5 \%-29.0 \%, 1 \%-29.5 \%$, and $0.5 \%-32 \%$ respectively. For the small road category, the Left, Center, and Right side error rate ranges are $0 \%-33.18 \%, 0 \%-26.82 \%$, and $1 \%-31.36 \%$ respectively. These error values are occured because the angle of image capturing is too small that it made the road looks smaller. Based on these errors, alley and small road category has width range between 1.72 to $2.28 \mathrm{~m}$ and 2.67 to $3.33 \mathrm{~m}$. Fig. 18 and 19, show the testing result of error rate for alley and small road category. 
Table 1. Sample of Error Rate for Alley and Small Road

\begin{tabular}{|c|c|c|c||c|c|c|c|}
\hline \multirow{2}{*}{ Sample Road } & \multicolumn{3}{|c||}{ Alley } & \multirow{2}{*}{ Sample Road } & \multicolumn{3}{|c|}{ Small Road } \\
\cline { 2 - 6 } & Left & Center & Right & & Left & Center & Right \\
\hline 1 & $2.73 \%$ & $9.09 \%$ & $7.27 \%$ & 1 & $4.33 \%$ & $0 \%$ & $10.67 \%$ \\
\hline 2 & $3.85 \%$ & $16.92 \%$ & $9.23 \%$ & 2 & $5 \%$ & $14 \%$ & $2.67 \%$ \\
\hline 3 & $3.33 \%$ & $6.11 \%$ & $15 \%$ & 3 & $5.67 \%$ & $16.67 \%$ & $11.33 \%$ \\
\hline 4 & $12.22 \%$ & $16.11 \%$ & $13.33 \%$ & 4 & $13 \%$ & $2.33 \%$ & $14.67 \%$ \\
\hline 5 & $7.65 \%$ & $8.24 \%$ & $1.18 \%$ & 5 & $5 \%$ & $0.33 \%$ & $1 \%$ \\
\hline
\end{tabular}

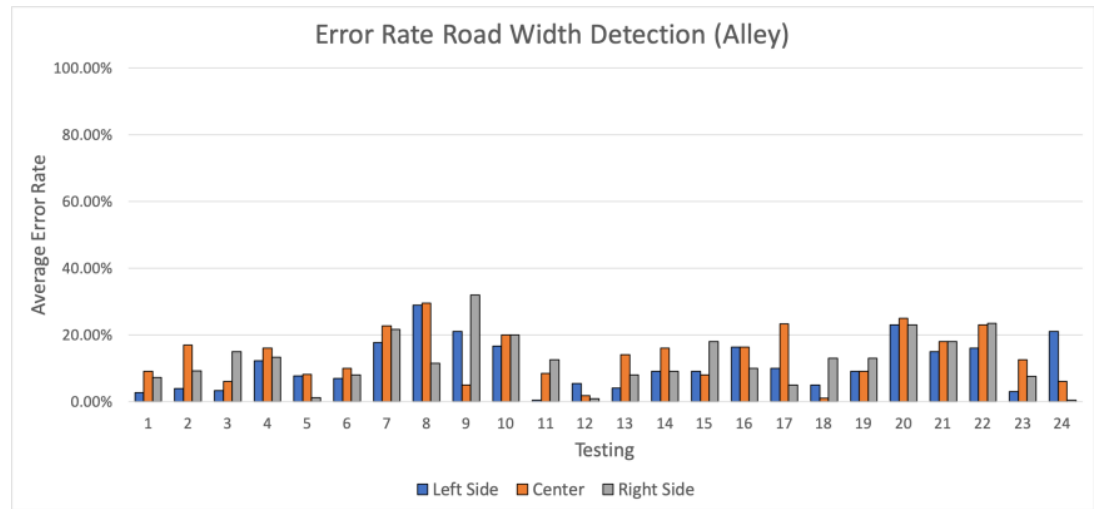

Fig. 18.Error Rate Road Width Detection for Alley

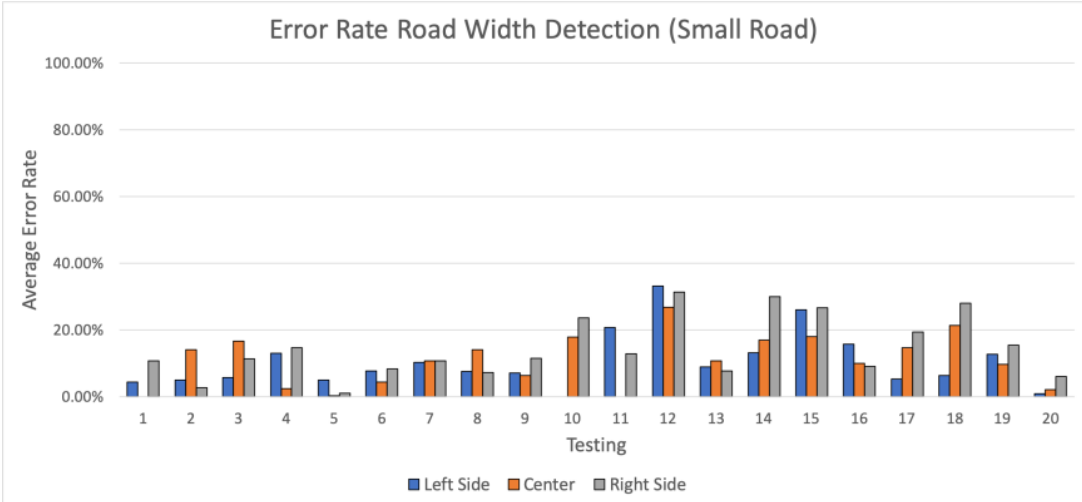

Fig. 19. Error Rate Road Width Detection for Small Road

Average error rate in alley category is acceptable, since alley is used only for motorcycle. Alley width prediction is in the range 1.72 to $2.28 \mathrm{~m}$, and its is in the range of motorcycle size. Small road category is also considered acceptable, since the usage of this type of road is also commonly used by motorcycle and its rarely used by car. 
Small road width prediction is in the range 2.67 to $3.33 \mathrm{~m}$ which just in the right size of a car.

Error rate for medium and big road: Table 2 shows some result of error rate for road with medium and big category. Average error rate for medium road category is between 9 to $11 \%$ with average error detection is $38-67 \mathrm{~cm}$. Fig. 20 shows that medium road category has highest error rate at $55.63 \%$ or deviation by $1.78 \mathrm{~m}$. This is because the capturing angle made the road looks bigger.

Table 2. Sample of Error Rate for Medium and Big Road

\begin{tabular}{|c|c|c|c|c|c|c|c|}
\hline \multirow{2}{*}{$\begin{array}{c}\text { Sample } \\
\text { Road }\end{array}$} & \multicolumn{3}{|c|}{ Medium Road } & \multirow{2}{*}{$\begin{array}{c}\text { Sample } \\
\text { Road }\end{array}$} & \multicolumn{3}{|c|}{ Big Road } \\
\hline & Left & Center & Right & & Left & Center & Right \\
\hline 1 & $5.94 \%$ & $0.31 \%$ & $2.19 \%$ & 1 & $13.17 \%$ & $4.15 \%$ & $39.27 \%$ \\
\hline 2 & $3.25 \%$ & $1.25 \%$ & $25 \%$ & 2 & $15.43 \%$ & $20.57 \%$ & $17.86 \%$ \\
\hline 3 & $5.76 \%$ & $0 \%$ & $4.85 \%$ & 3 & $19.47 \%$ & $28.42 \%$ & $0 \%$ \\
\hline 4 & $2 \%$ & $0 \%$ & $6.57 \%$ & 4 & $2.73 \%$ & $20.91 \%$ & $24.73 \%$ \\
\hline 5 & $6.25 \%$ & $7.25 \%$ & $12.5 \%$ & 5 & $1.83 \%$ & $25.17 \%$ & $12 \%$ \\
\hline
\end{tabular}

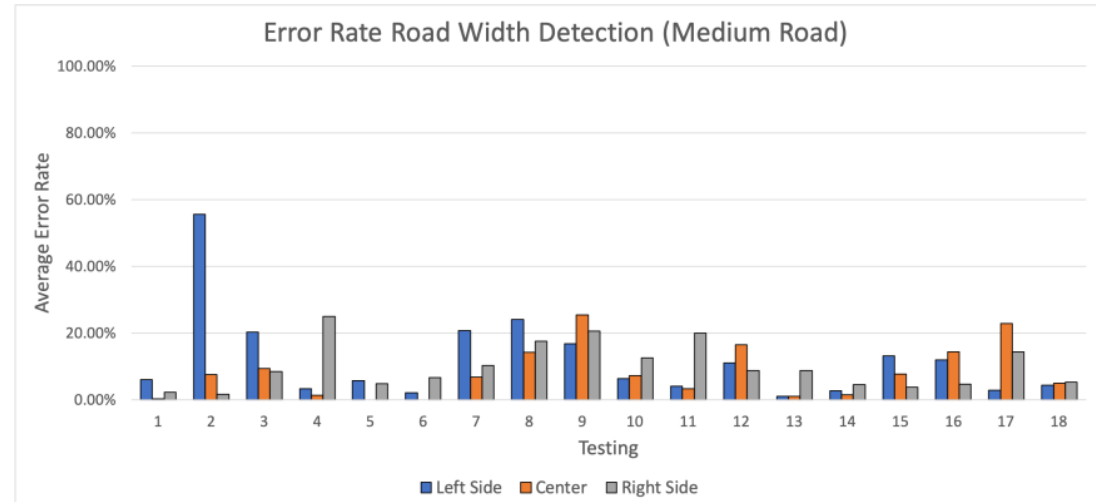

Fig. 20. Error Rate Road Width Detection for Medium Road

Testing for big road requires a lot of normalization method, as mentioned in equation 4 and 5. The road looks smaller due to the effect of perspective image and vanishing point higher than another road category. Normalization method could make error rate smaller than before, but it could not be avoided because the comparative value of road is too high. Fig. 21 shows big road category has highest error rate at $47.33 \%, 36 \%$, and $39.27 \%$. 


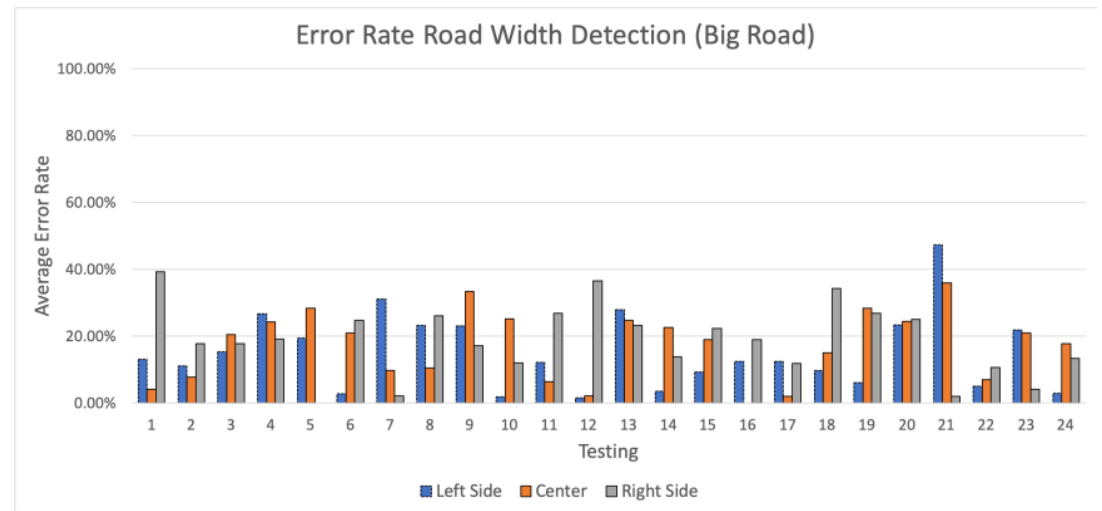

Fig. 21. Error Rate Road Width Detection for Big Road

Based on its calculation, medium road's width has range between 2.61 to $4.52 \mathrm{~m}$ and meanwhile, big road category has range 3.2 to $4.8 \mathrm{~m}$. Eventhough had high error rate, medium and big road category is acceptable since this type of roads is used by every kind of vehicles.

Average error rate road width: Table 3 shows the result of testing from every road category and capture angle (left, center, and right side). Those average error rate for every road in each side are in the range of $10-15 \%, 8-18 \%$, and $10-19 \%$ for left, center, and right side respectively. The maximum distance difference between width of the road and the detection result in big road category is $3.55 \mathrm{~m}$.

Table 3. Result of Average Error Rate for Every Category and Sides

\begin{tabular}{|l|c|c|c|}
\hline \multicolumn{1}{|c|}{ Road Category } & Left Side & Center & Right Side \\
\hline Alley & $10.35 \%$ & $13.03 \%$ & $12.35 \%$ \\
\hline Small Road & $10.11 \%$ & $10.07 \%$ & $13.11 \%$ \\
\hline Medium Road & $12.84 \%$ & $7.85 \%$ & $10.34 \%$ \\
\hline Big Road & $15.18 \%$ & $17.90 \%$ & $19.41 \%$ \\
\hline
\end{tabular}

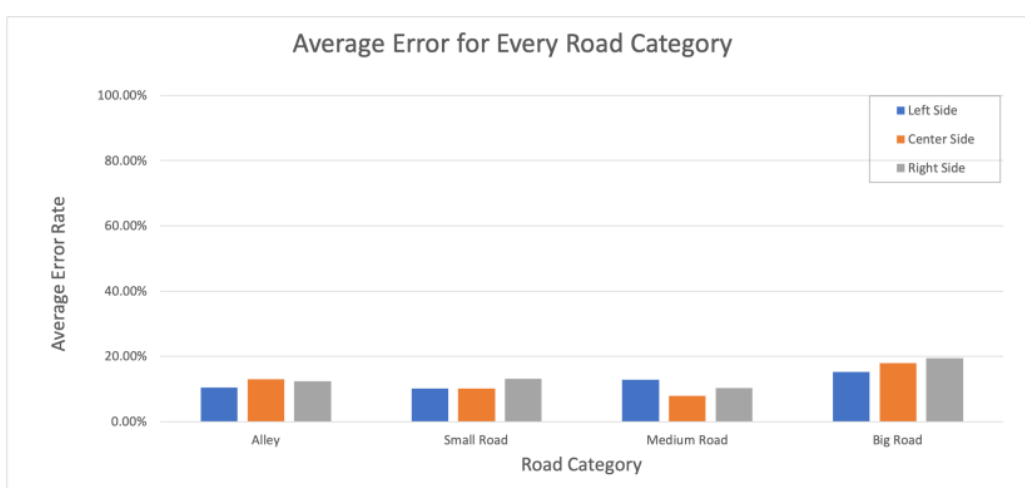

Fig. 22. Average Error for Every Road Category 
Based on these results, lowest error rate for every type of roads is taken from left sides with has range between $10 \%$ to $15 \%$. Bigger roads make higher error rate due to the effect of perspective, thus make pixel/meter comparation became less accurate.

\subsection{Road category classification}

Average error rate for every road category is used to reclassify a road. Every road category has new upper and lower bound based on its average error rate. By using using algorithm in Fig. 23, the new upper and lower bound is defined.

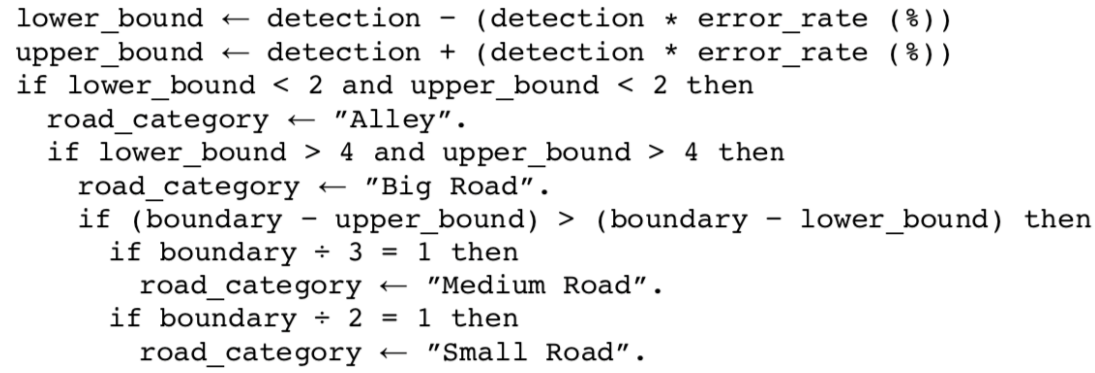

Fig. 23. Road Category Classification Algorithm

Reclassification is done by matching road with new bounds and it will be compared with the old one. The results of reclassification testing are $70.93 \%, 73.26 \%$, and $75.58 \%$ for left, center, and right sides of capturing angle. Fig. 24 shows the accuracy of road reclassification. These results show that the method that proposed is reliable for every sides of the road.

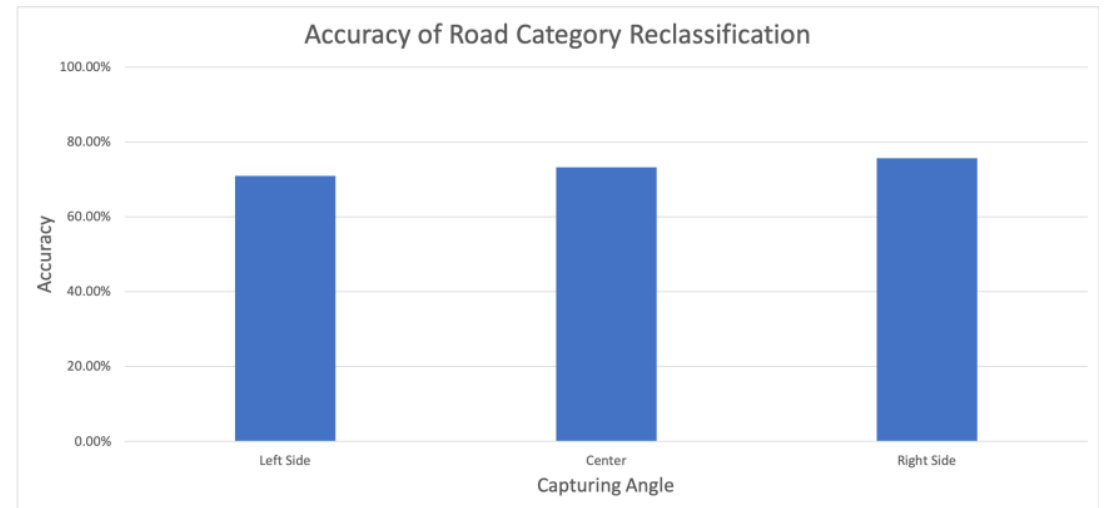

Fig. 24. Accuracy of Road Category Reclassification 


\section{$5 \quad$ Conclusion and Future Works}

The proposed system aims to create an application to record road situation. It is designed for Android smartphone, and it worked well according that was designed. Road information will be collected more when this application is run by many drivers.

In this paper, the collected data was processed using object and lane detection in order to calculate road width (when lane boundaries exist), or using three vanishing points to create a virtual lane on captured images (when lane boundaries do not exist). Road width calculation is done by measuring the distance between two intersection points in designated height coordinates. This road width measurement is important for checking the suitability of an alternate road to a certain type of vehicle based on its size (e.g. small car or motorcycle).

Average error rate for road width measurement using object and lane detection (lane boundaries exist) is $19.71 \%$, while the average error rate for road without boundary is in range of $10-15 \%, 8-18 \%$, and $10-19 \%$ for measurement from left, center and right sides of the road respectively. This error value is getting higher along with road width, and it happens due to the effect of perspective image made pixel/meter comparation is not too accurate. Accuracy of road category reclassification to define road category is $70.93 \%, 73.26 \%$, and $75.58 \%$ for left, center, and right sides of road respectively, and it is reliable for every side in the road.

Our results show that our proposed system is acceptable and feasible, since it suits the type of vehicles using respective road categories. Alley and small road category are commonly used by motorcycle, while other road categories can be used by all type of vehicle in Indonesia. Other roads have the range of $2.61 \mathrm{~m}$ to $4.8 \mathrm{~m}$, and it is suitable for common cars.

Further works can be conducted to improve the normalization system for reducing errors, especially for medium and big road categories. Another matter that can be addressed is to have extended and more practical traffic scenarios. Also, in order to achieve more valid and real data, crowd collecting system can be used as well.

\section{References}

[1] Hu, W., Yuanlin Liu, Li Li, and Shujie Xin. (2010). The Short-Term Traffic Flow Prediction Based on Neural Network, 2nd International Conference on Future Computer and Communication. https://doi.org/10.1109/icfcc.2010.5497785

[2] Lubis, H. A.-R. S. (2009). Motorcycles Growth and Its Impacts to Urban Transportation, Proceedings of the Eastern Asia Society for Transportation Studies, 7, 329.

[3] Nasution, S.M., Husni, E. and Kuspriyanto. (2018). The Effect of Heterogeneous Traffic Flow on The Transportation System, International Conference on Electrical Engineering and Computer Science, Bali, Indonesia.

[4] Barmpounakis, E. N., Vlahogianni, E. I. and Golias, J. C. (2016). Intelligent Transportation Systems and Powered Two Wheelers Traffic. IEEE Transactions on Intelligent Transportation Systems, 17(4), 908. https://doi.org/10.1109/tits.2015.2497406 
[5] Minh, C. C., Matsumoto, S. and Sano, K. (2005). The Speed, Flow, and Headway Analyses of Motorcycle Traffic. Journal of the Eastern Asia Society for Transportation Studies, 6, $1496-1508$.

[6] Papadakis, S. and Kalogiannakis, M. (2017) 'Mobile Educational Applications for Children: What Educators and Parents Need to Know', Int. J. Mobile Learning and Organisation, Vol. 11, No. 3, pp.256-277. https://doi.org/10.1504/ijmlo.2017.085338

[7] Hoang, T.M., Nguyen, P.H., Truong, N.Q., Lee, Y.W., and Park, K.R. (2019). Deep RetinaNet-Based Detection and Classification of Road Markings by Visible Light Camera Sensors. Sensors 2019, 19, 281. https://doi.org/10.3390/s19020281

[8] Wang, Hai, Yijie Yu, Yingfeng Cai, Xiaobo Chen, Long Chen, and Qingchao Liu. (2019). A Comparative Study of State-of-the-Art Deep Learning Algorithms for Vehicle Detection. IEEE Intelligent Transportation Systems Magazine: Summer 2019. https://doi.org/ $10.1109 /$ mits.2019.2903518

[9] Yan, X., and Yanqiang Li. (2017). A Method of Lane Edge Detection Based on Canny Algorithm, Chinese Automation Congress (CAC), Jinan, China. https://doi.org/10.1109/cac. 2017.8243122

[10] Acharya, A.J and Sheetal Thakar. (2017). Road Lane and Edge Detection with Gradient and Hough Transform. International Education and Research Journal, 3(5).

[11] Samuel, M., Maziah Mohamad, Shaharil Mad Saad, and Mohamed Hussein. (2018). Development of Edge-Based Lane Detection Algorithm Using Image Processing. International Journal on Informatics Visualization, 2(1). https://doi.org/10.30630/joiv.2.1.101

[12] Rajkumar, C. and S.K. Mahendran. (2017). Edge Detection for Night Vision Images Using Canny. International Journal of Advanced Research in Computer Science \& Technology, $5(1)$.

[13] Mu, C. and Xing Ma. (2014). Lane Detection Based on Object Segmentation and Piecewise Fitting. Telkomnika Indonesian Journal of Electrical Engineering, 12(5). https://doi. org/10.11591/telkomnika.v12i5.3240

[14] Liu, G., Li, S. and Liu, W. (2013). Lane Detection Algorithm Based on Local Feature Extraction. 2013 Chinese Automation Congress, Changsha, China. https://doi.org/10.1109/ cac. 2013.6775702

[15] Andrade, D. C., Bueno, F., Franco, F. R., Silva, R. A., Neme, J. H. Z., Margraf, E., Omoto, W. T., Farinelli, F. A., Tusset, A. M., Okida, S., Santos, M. M. D., Ventura, A., Carvalho, S. and dos Santos Amaral, R. (2019). A Novel Strategy for Road Lane Detection and Tracking Based on a Vehicle's Forward Monocular Camera, IEEE Transactions on Intelligent Transportation Systems, 20(4). https://doi.org/10.1109/tits.2018.2856361

[16] Kluger F., Ackermann H., Yang M.Y., Rosenhahn B. (2017). Deep Learning for Vanishing Point Detection Using an Inverse Gnomonic Projection. In: Roth V., Vetter T. (eds) Pattern Recognition. GCPR 2017. Lecture Notes in Computer Science, vol 10496. Springer, Cham. https://doi.org/10.1007/978-3-319-66709-6_2

[17] Shi, J., Jinxiang Wang, and Fangfa Fu. (2016). Fast and Robust Vanishing Point Detection for Unstructured Road Following. IEEE Transactions on Intelligent Transportation Systems, 17(4), 970. https://doi.org/10.1109/tits.2015.2490556

\section{Authors}

Surya Michrandi Nasution graduated from IT Telkom Bandung in Bachelor of Telecommunication Engineering in 2009 and Master Degree of Electrical Engineering in 2012 from Institut Teknologi Bandung. Currently, he is a researcher at Institut 
Teknologi Bandung. His research interest lies in the area of Internet of Things, Mobile Computing, Intelligent Transportation System, and Security. (e-mail: surya.michrandi@gmail.com)

Emir Husni is a Professor at School of Electrical Engineering \& Informatics at Institut Teknologi Bandung. Husni received the Ir. degree in Electrical Engineering from Institut Teknologi Bandung, the M.Sc. and Ph.D. degrees in Satellite Communication and Satellite Technology from University of Surrey, UK. Husni's research interests are in satellite technology, internet of things, and pervasive computing. (email: ehusni@1skk.ee.itb.ac.id).

Kuspriyanto is a Professor at the School of Electrical Engineering and Informatics, Institut Teknologi Bandung. He received his bachelor degree in Electrical Engineering from Institut Teknologi Bandung in 1974, D.E.A (1979) and Ph.D. (1981) in Automatic System from USTL, France. His field of interest includes Computer System, Computer Architecture, and Real Time Systems. (e-mail: kuspriyanto@ yahoo.com)

Rahadian Yusuf received his bachelor and master degrees in Electrical and Informatics Engineering from Bandung Institute of Technology and Ph.D. of Engineering degree from Doshisha University, in 2005, 2011, and 2017, respectively. In 2017, he joined Doshisha University, Japan. In 2019 he joined ITB, Indonesia. His research interests include affective computing, human-system interaction, evolutionary systems, and socio-informatics.

Rahmat Mulyawan received his B.Sc. in EE from ITB (Indonesia) in 2008 and M.Sc. in EE from TU Delft (the Netherlands) in 2011. He is a lecturer in the School of Electrical Engineering and Informatics, Bandung Institute of Technology. His research interests include intelligent mobility systems, communication system transceiver design, and system optimisation for IoT applications. 